\title{
Intravoxel Incoherent Motion Diffusion Weighted Imaging for the Therapeutic Response of Transarterial Chemoembolization for Hepatocellular Carcinoma
}

\author{
Juan Peng1,2, Cui Yang1, Jing Zheng1, Ran Wang1, Yi Zhou', Weicheng Wang1, Lin Yang1,

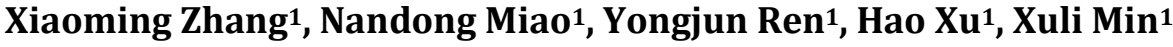 \\ ${ }^{1}$ Sichuan Key Laboratory of Medical Imaging, Department of Radiology, Affiliated Hospital of North Sichuan Medical College, \\ Nanchong, China \\ ${ }^{2}$ Department of Radiology, Sichuan Provincial People’s Hospital Jinniu Hospital, Chengdu, China \\ Email: linyangmd@163.com
}

How to cite this paper: Peng, J., Yang, C., Zheng, J., Wang, R., Zhou, Y., Wang, W.C., Yang, L., Zhang, X.M., Miao, N.D., Ren, Y.J., Xu, H. and Min, X.L. (2019) Intravoxel Incoherent Motion Diffusion Weighted Imaging for the Therapeutic Response of Transarterial Chemoembolization for $\mathrm{He}$ patocellular Carcinoma. Journal of Cancer Therapy, 10, 591-601. https://doi.org/10.4236/jct.2019.107048

Received: June 25, 2019

Accepted: July 21, 2019

Published: July 24, 2019

Copyright $\odot 2019$ by author(s) and Scientific Research Publishing Inc. This work is licensed under the Creative Commons Attribution International License (CC BY 4.0).

http://creativecommons.org/licenses/by/4.0/

\section{(c) (i) Open Access}

\begin{abstract}
Background: Intravoxel incoherent motion diffusion weighted imaging (IVIM-DWI) can not only observe the diffusion of tissue water molecules but also reflect the blood perfusion information of tissue microcirculation. IVIM-DWI has been applied in many clinical areas. However, few studies have addressed the use of IVIM-DWI for the evaluation of transarterial chemoembolization (TACE) response in hepatocellular carcinoma (HCC) patients. Objectives: The purpose of the present study was to explore the role of IVIM-DWI for the therapeutic response of TACE for HCC. Materials and Methods: Twenty patients underwent IVIM-DWI scan on a 3.0T magnetic resonance imaging instrument $1-3$ days before and 30 to 40 days after TACE. The values of IVIM-DWI parameters, including standard apparent diffusion coefficient (ADC), pure diffusion coefficient (Dslow), pseudo-diffusion coefficient (Dfast) and perfusion fraction $(f)$ were measured. The values of IVIM-DWI parameters before and after TACE were compared using paired $\mathrm{t}$ tests. The values between responsive and non-responsive groups were compared using independent-samples $\mathrm{t}$ test. $\mathrm{P}<0.05$ indicated statistical significance. Results: After TACE, the ADC and Dslow values in the tumors increased significantly, and the values of Dfast decreased significantly, while the values of $f$ value did not change obviously. The $\mathrm{ADC}$ values in responsive group were remarkably higher than those in non-responsive group, the Dfast values in responsive group were significantly lower than those in non-responsive group, but the values of Dslow and $f$ between the two groups were not different significantly. Conclusions: IVIM-DWI parameters can be used as potential
\end{abstract}


markers for the therapeutic response of TACE for HCC.

\section{Keywords}

Hepatocellular Carcinoma, Diffusion-Weighted Imaging, Intravoxel Incoherent Motion, Transarterial Chemoembolization

\section{Introduction}

Hepatocellular carcinoma (HCC) is one of the leading causes of cancer deaths worldwide and is a serious threat to human life and health. Because HCC often has an occlusive onset, patients are often in the advanced stages of the disease when first seeking treatment and have lost the indications for surgical excision. Transarterial chemoembolization (TACE) has been proven to improve the survival time and life quality in HCC patients [1] [2] [3] [4] [5]. The accurate evaluation of TACE treatment response is conducive to the next step of developing personalized treatment programs. However, assessment of anatomic response in the early post-treatment period has been controversial because the absence of a reduction in tumor size does not mean an absence of response [6] [7] [8]. Magnetic resonance diffusion weighted imaging (DWI) can detect the free diffusion motion of water molecules in living tissue. It enables quantitative analyses by measuring the standard apparent diffusion coefficient (ADC) value. Thus, this technique can be used to evaluate TACE response for HCC [9]-[14]. But conventional DWI with a mono-exponential model could not separate perfusion and true diffusion-related effect. Intravoxel incoherent motion diffusion weighted imaging (IVIM-DWI), with a bi-exponential model, can not only observe the diffusion of tissue water molecules but also reflect the blood perfusion information of tissue microcirculation, compensating for the deficiencies of conventional DWI [15]. In recent years, IVIM-DWI has been applied in many clinical areas [16]-[26]. However, few studies have addressed the use of IVIM-DWI for the therapeutic response of TACE for HCC. The aim of the present study was to investigate the role of IVIM-DWI for the therapeutic response of TACE for HCC.

\section{Materials and Methods}

\subsection{Patient Population}

This study was approved by our institutional review board, and patient informed consent was obtained. In this study, TACE was performed in the intermediate-stage HCC patients who were not suitable candidates for surgical resection. Exclusion criteria were advanced-stage HCC in the BCLC classification, serum total bilirubin $>80 \mathrm{umol} / \mathrm{L}$, history of severe heart or renal impairment, and iodine allergy. From January 2015 to December 2016, 20 patients with 31 HCC nodules were consecutively enrolled in this study, including 16 males and 4 fe- 
males, aged $24-72$ years, with an average age of $54.3 \pm 10.4$ years (Table 1 ). All patients had not received other antitumor treatments before receiving TACE treatment. HCCs were classified as either response group or non-response group according to morphological features. The HCC nodules with diameter reduction greater than $30 \%$ constituted the response group, nodules with diameter reduction less than $30 \%$ or increment constituted the non-response group.

\subsection{MR Imaging}

All patients received MRI examinations at 1 - 3 days before TACE and $30-40$ days after TACE. The equipment used was a 3.0T magnetic resonance imaging (MRI) scanner (Discovery MR750, GE Medical Systems, Milwaukee, Wis., USA). Patients fasted for 4 hours and received breathing training before scanning. The scanning range was the full liver, from the top of the diaphragm to the lower edge of the liver. The scanning sequences were a breath-hold transverse axis fat-suppressed T1WI sequence, a respiratory-triggered fat-suppressed T2WI, and an IVIM-DWI $(\mathrm{b}=0,20,40,80,100,200,400,800,1000)$ with a TR $3529 \mathrm{~ms}$, TE $60.8 \mathrm{~ms}$, matrix $128 \times 160$, FOV $40 \mathrm{~cm}$, slice thickness $5 \mathrm{~mm}$, and interslice gap $0.5 \mathrm{~mm}$, respectively. Images were transferred to a GE ADW4.6 post-processing station and analyzed using Function-MADC software. The ADC, pure diffusion coefficient (Dslow), pseudodiffusion coefficient (Dfast) and perfusion fraction $(f)$ values were measured for each patient. Various parameters were measured 3 times, and the average was taken.

\subsection{TACE Procedure}

The Seldinger technique is commonly used to puncture the right femoral artery. Under the guidance of a guide wire, a $5 \mathrm{~F}$ angiographic catheter was placed in the celiac artery or hepatic artery for the performance of digital subtraction angiography (DSA). Subsequently, microcatheter superselective catheterization of the tumor-feeding artery was conducted. TACE was performed by administration of 5-fluorouracil (1000 mg) via the tumor-feeding artery, followed by lipiodol (Lipiodol UltraFluid, Laboratoire Guerbet, Aulnay-Sous-Bois, France) (5 to $20 \mathrm{ml}$ )

Table 1. Clinical characteristics of the 20 patients.

\begin{tabular}{lc}
\multicolumn{1}{c}{ Variable } & Value \\
\hline Age $(\mathrm{yr})$ & 54.3 (range $24-72)$ \\
Sex $\quad$ Men & 16 \\
Women & 4.4 (range $2.5-8.9)$ \\
Size of tumor $(\mathrm{cm})$ & 19 \\
Liver cirrhosis & 19 \\
HBV & $14,082.1$ (range $665.5-110,980.7)$ \\
Serum AFP $(\mathrm{ng} / \mathrm{mL})$ &
\end{tabular}

HBV: Hepatitis B virus; AFP: alpha-fetoprotein. 
with lobaplatin $(40 \mathrm{mg})$ and adriamycin $(40 \mathrm{mg})$ mixed chemoembolic emulsion and gelfoam particles. Chemobolization was terminated when the tumor stain disappeared or when the patient could no longer tolerate the procedure.

\subsection{Statistical Analysis}

SPSS 20.0 software was used to analyze the changes of IVIM-DWI parameters. The IVIM-DWI parameters of HCC nodules before and after TACE were compared using paired $t$ test. The values between responsive and non-responsive groups were compared using independent-samples $\mathrm{t}$ test. $\mathrm{P}<0.05$ indicated statistical significance.

\section{Results}

After treatment with TACE, the ADC and Dslow values in the tumor increased remarkably (all $\mathrm{p}<0.01$ ), and the values of Dfast decreased significantly ( $\mathrm{p}<$ $0.01)$, while the values of $f$ did not change obviously $(p>0.05)$. Six HCC nodules were classified as responsive group, and 25 HCC nodules were non-responsive group. The ADC values in responsive group were evidently higher than those in non-responsive group $(\mathrm{p}<0.05)$, the Dfast values in responsive group were significantly lower than those in non-responsive group $(p<0.05)$, but the values of Dslow and $\mathrm{f}$ between the two groups were not different significantly after TACE (all p > 0.05) (Table $2 \&$ Table 3) (Figure $1 \&$ Figure 2). The follow-up period was 1.6 to 3 months. TACE related complications and perioperative deaths have not occurred.

Table 2. Comparison of IVIM-DWI parameter levels before and after TACE treatment.

\begin{tabular}{ccccc}
\hline Parameters & Pre-TACE & Post-TACE & $\mathrm{t}$ & $\mathrm{p}$ \\
\hline ADC $\left(10^{-3} \mathrm{~mm}^{2} / \mathrm{s}\right)$ & $0.94 \pm 0.17$ & $1.23 \pm 0.21$ & 8.794 & $<0.01$ \\
Dslow $\left(10^{-3} \mathrm{~mm}^{2} / \mathrm{s}\right)$ & $0.72 \pm 0.14$ & $0.91 \pm 0.14$ & 6.120 & $<0.01$ \\
Dfast $\left(10^{-3} \mathrm{~mm}^{2} / \mathrm{s}\right)$ & $37.14 \pm 7.96$ & $28.29 \pm 7.24$ & 5.612 & $<0.01$ \\
$\mathrm{f}(\%)$ & $15.52 \pm 3.33$ & $15.45 \pm 3.84$ & 0.091 & 0.928 \\
\hline
\end{tabular}

IVIM-DWI: intravoxel incoherent motion diffusion weighted imaging; TACE: transarterial chemoembolization; ADC: apparent diffusion coefficient; Dslow: pure diffusion coefficient; Dfast: pseudodiffusion coefficient; $f$ : perfusion fraction.

Table 3. Comparison of IVIM-DWI parameter levels after TACE between responsive group and non-responsive group.

\begin{tabular}{ccccc}
\hline Parameters & Responsive group & Non-responsive group & $\mathrm{t}$ & $\mathrm{p}$ \\
\hline ADC $\left(10^{-3} \mathrm{~mm}^{2 / \mathrm{s}}\right)$ & $1.42 \pm 0.13$ & $1.18 \pm 0.19$ & 2.769 & 0.010 \\
Dslow $\left(10^{-3} \mathrm{~mm}^{2} / \mathrm{s}\right)$ & $0.99 \pm 0.10$ & $0.87 \pm 0.14$ & 1.961 & 0.059 \\
Dfast $\left(10^{-3} \mathrm{~mm}^{2} / \mathrm{s}\right)$ & $22.16 \pm 4.39$ & $29.75 \pm 7.06$ & 2.506 & 0.018 \\
$\mathrm{f}(\%)$ & $14.88 \pm 3.22$ & $15.58 \pm 4.03$ & 0.389 & 0.700 \\
\hline
\end{tabular}

IVIM-DWI: intravoxel incoherent motion diffusion weighted imaging; TACE: transarterial chemoembolization; ADC: apparent diffusion coefficient; Dslow: pure diffusion coefficient; Dfast: pseudodiffusion coefficient; $f$ : perfusion fraction. 


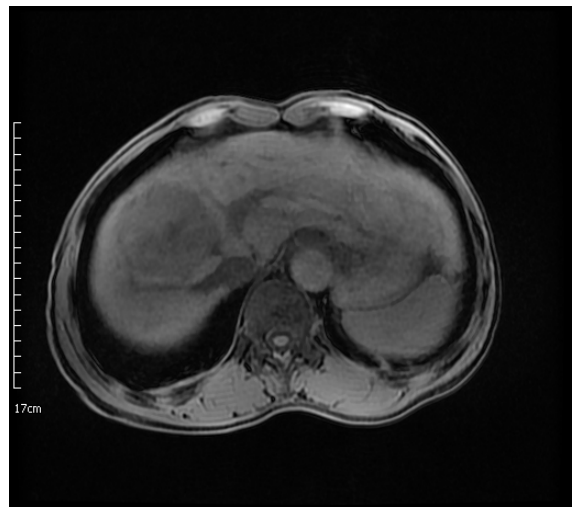

(a)

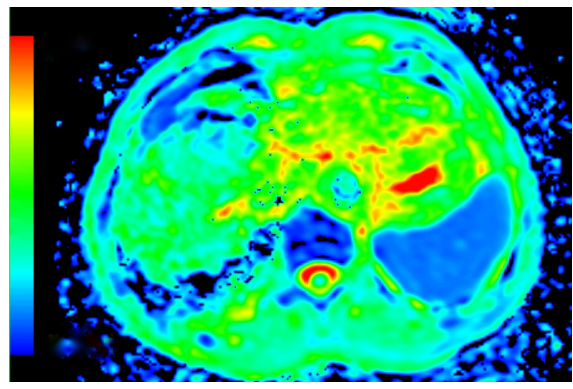

(c)

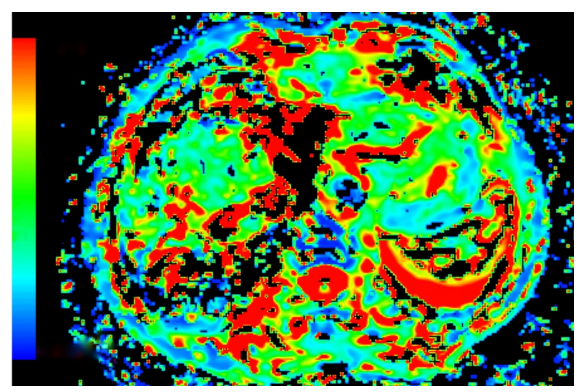

(e)

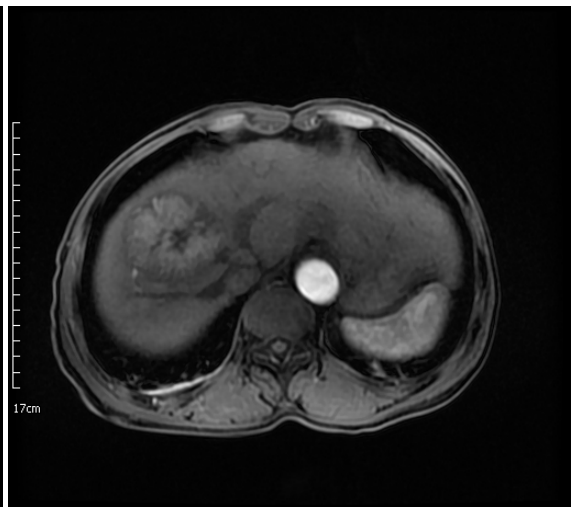

(b)

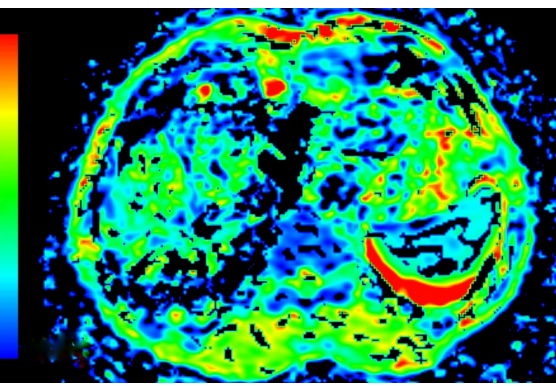

(d)

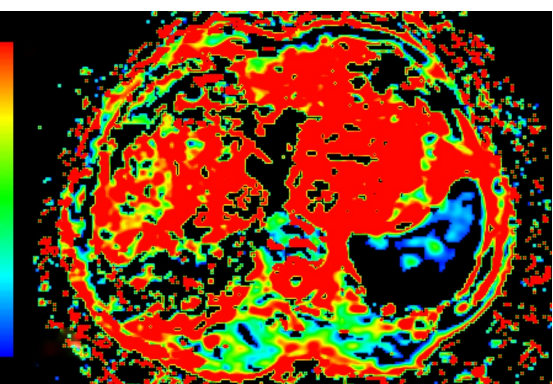

(f)

Figure 1. Axial MRI images of HCC patient before TACE treatment. (a) T1-weighted unenhanced image shows a hypointensive lesion in right lobe of liver. (b) Arterial phase contrast-enhanced image shows a heterogeneous enhanceed lesion. (c) ADC map. (d) Dslow map. (e) Dfast map. (f) f map.

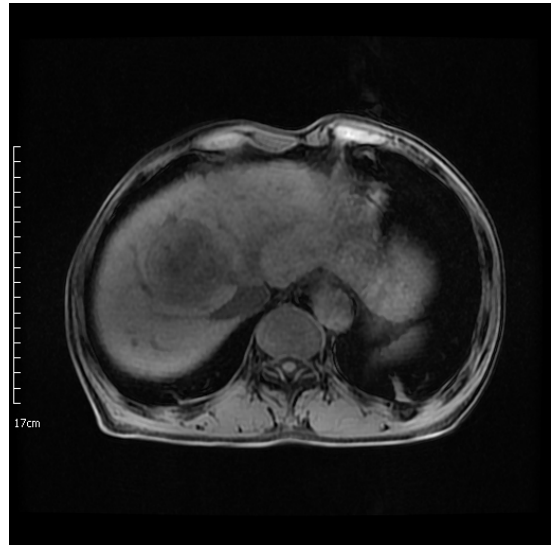

(a)

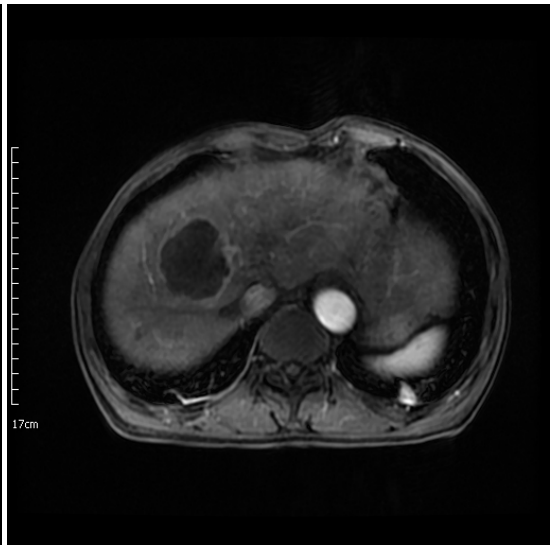

(b) 


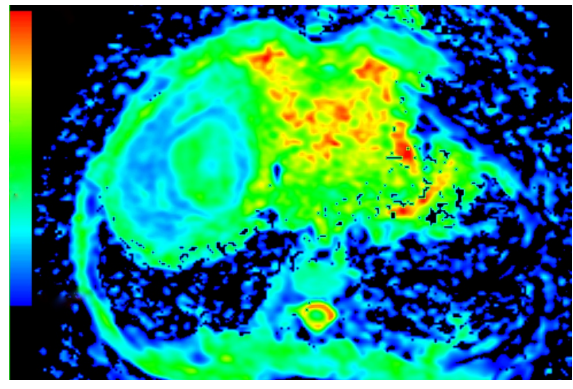

(c)

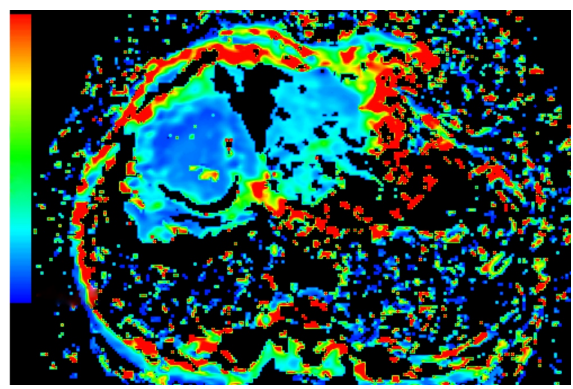

(e)

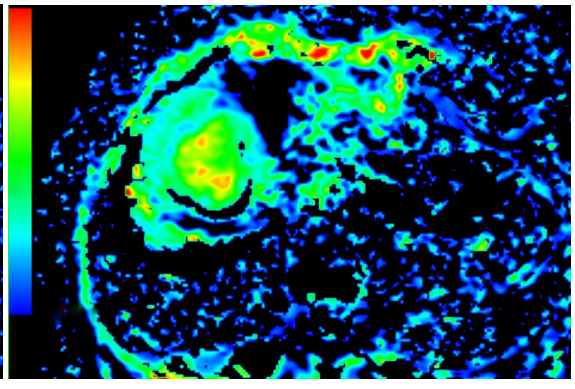

(d)

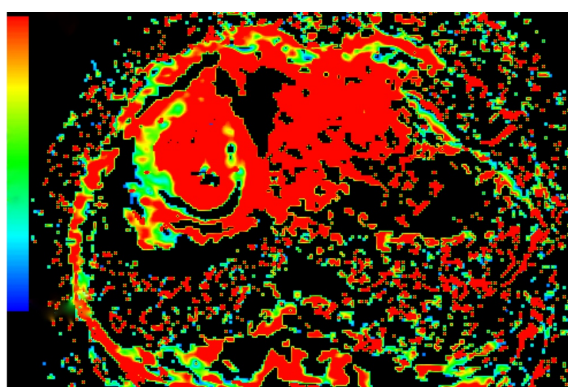

(f)

Figure 2. Axial MRI images of the same patient as in Figure 1 after TACE treatment. (a) T1-weighted unenhanced image shows a hypointensive lesion in right lobe of liver. (b) Arterial phase contrast-enhanced image shows the central necrotic area and peripheral enhanced area in the lesion. (c) ADC map. (d) Dslow map. (e) Dfast map. (f) f map.

\section{Discussion}

Therapeutic response assessment of TACE is crucial for selecting the next treatment regimen and prognosis evaluation. Serun biomarkers often be used as indicators for the diagnosis and monitoring of tumor treatment progress [27] [28] [29]. Serum alpha-fetoprotein (AFP) is one of the most commonly used indicators for evaluating the response of TACE treatment. However, AFP values are always at normal levels in 30\% of HCC patients [30]. For this group of patients, AFP values cannot be used to evaluate TACE response. Medical imaging plays an important role in response evaluation of tumor therapy [6] [31] [32] [33] [34] [35]. Currently, the value of functional imaging for tumor therapeutic response evaluation is increasingly being recognized [31]. Computed tomography (CT) can clearly show the deposition of high-density iodized oil in a lesion. However, the high-density iodized oil in the lesion interferes with the observation of residual tumor via enhanced CT. CT perfusion imaging has the ability to assess the perfusion changes in HCC tissues after chemoembolization, which can be used to evaluate the treatment response of TACE for HCC, but it will increase radiation exposure [6] [31].

With the development of functional MRI, it is possible to determine the metabolic status of tumor cells at an early stage before the appearance of tumor-related morphological changes. IVIM-DWI can show the molecular diffusion and microperfusion of the local capillary network in lesions by using quantitative indicators [15]. The commonly used parameters include the Dslow, Dfast and $f$ values. The Dslow parameter of IVIM-DWI removes the effects of microcirculation 
perfusion on ADC and can more accurately reflect the diffusion of water molecules within tissue. Dfast and $\mathrm{f}$ values mainly reflect tissue microcirculation capillary perfusion.

Park YS et al. studied 44 HCC patients using IVIM-DWI prior to TACE and CT after TACE as a reference standard. In total, 51 HCCs were analyzed: 37 were lipiodol good uptake (LGU) group and 14 were lipiodol poor uptake (LPU) group. $\mathrm{ADC}, \mathrm{D}$, and $\mathrm{f}$ values were not significantly different between these two groups. $\mathrm{D}^{*}$ was significantly elevated in LGU than LPU. Their result indicated that the IVIM-DWI parameters would be helpful for predicting the lipiodol uptake [36]. Wu L et al. explore the threshold of IVIM parameters, ADC ratios 24 48 hours after TACE to assess early response in patients with unresectable HCC. They found that the ADC ratio and D ratio after TACE were independent predictors for response to TACE for HCC, and showed stronger association with PFS than mRECIST [37].

In this study, the values of ADC and Dslow of lesions after TACE treatment increased significantly, which may be related to the fact that the tumor cells undergo necrosis, leading to increased water diffusion capacity. The Dfast values in the lesion areas of HCC patients decreased after TACE, compared with the before-treatment values, which may be related to the fact that tumor blood supplies were blocked after TACE and the basement membrane of newly generated blood vessels was incomplete and the vessels had a small diameter, leading to slow blood flow. The value of $f$ in this study did not change significantly after treatment, likely because Dfast and $\mathrm{f}$ values represent different aspects of perfusion; the former is primarily related to local tissue capillary velocity, and the latter is related to local tissue blood volume [36].

This study has the following limitations. Firstly, the number of cases collected in this study is small. Secondly, the images analyzed before and after TACE treatment may not be the same levels, which may be a potential source of error. Another limitation is that the relationship between the prognosis and changes of IVIM parameters after TACE treatment was not investigated in the HCC patients. In further studies, an increased number of cases should be studied. The prognosis of the patients with HCC should be followed up. In addition, because IVIM parameter measurement is susceptible to the $b$ value and the selection of the $b$ value is still controversial [38] [39], the selection of the $b$ value needs to be further optimized or standardized.

\section{Conclusion}

In summary, IVIM-DWI parameters can be used as potential markers for the therapeutic response of TACE for HCC.

\section{Funding}

The study was supported by the Projects of Department of Science and Technology of Sichuan Province (2016JY0105). 


\section{Conflicts of Interest}

The authors have no conflicts of interest to declare.

\section{References}

[1] Wáng, Y.X., De Baere, T., Idée, J.M., et al. (2015) Transcatheter Embolization Therapy in Liver Cancer: An Update of Clinical Evidences. Chinese Journal of Cancer Research, 27, 96-121.

[2] Lo, C.M., Ngan, H., Tso, W.K., et al. (2002) Randomized Controlled Trial of Transarterial Lipiodol Chemoembolization for Unresectable Hepatocellular Carcinoma. Hepatology, 35, 1164-1171. https://doi.org/10.1053/jhep.2002.33156

[3] Llovet, J.M., Real, M.I., Montaña, X., et al. (2002) Arterial Embolisation or Chemoembolisation versus Symptomatic Treatment in Patients with Unresectable Hepatocellular Carcinoma: A Randomised Controlled Trial. The Lancet, 359, 1734-1739. https://doi.org/10.1016/S0140-6736(02)08649-X

[4] Han, K. and Kim, J.H. (2015) Transarterial Chemoembolization in Hepatocellular Carcinoma Treatment: Barcelona Clinic Liver Cancer Staging System. World Journal of Gastroenterology, 21, 10327-10335. https://doi.org/10.3748/wjg.v21.i36.10327

[5] Song, D.S., Nam, S.W., Bae, S.H., et al. (2015) Outcome of Transarterial Chemoembolization-Based Multi-Modal Treatment in Patients with Unresectable Hepatocellular Carcinoma. World Journal of Gastroenterology, 21, 2395-2404. https://doi.org/10.3748/wjg.v21.i8.2395

[6] Yang, L., Zhang, X.M., Tan, B.X., et al. (2012) Computed Tomographic Perfusion Imaging for the Therapeutic Response of Chemoembolization for Hepatocellular Carcinoma. Journal of Computer Assisted Tomography, 36, 226-230.

https://doi.org/10.1097/RCT.0b013e318245c23c

[7] Kamel, I.R., Liapi, E., Reyes, D.K., et al. (2009) Unresectable Hepatocellular Carcinoma: Serial Early Vascular and Cellular Changes after Transarterial Chemoembolization as Detected with MR Imaging. Radiology, 250, 466-473. https://doi.org/10.1148/radiol.2502072222

[8] Bruix, J., Sherman, M., Llovet, J.M., et al. (2001) Clinical Management of Hepatocellular Carcinoma. Conclusions of the Barcelona-2000 EASL Conference. European Association for the Study of the Liver. Journal of Hepatology, 35, 421-430. https://doi.org/10.1016/S0168-8278(01)00130-1

[9] Wu, X.M., Wang, J.F., Ji, J.S., et al. (2017) Evaluation of Efficacy of Transcatheter Arterial Chemoembolization for Hepatocellular Carcinoma Using Magnetic Resonance Diffusion-Weighted Imaging. OncoTargets and Therapy, 10, 1637-1643. https://doi.org/10.2147/OTT.S115568

[10] Lin, M., Tian, M.M., Zhang, W.P., et al. (2016) Predictive Values of Diffusion-Weighted Imaging and Perfusion-Weighted Imaging in Evaluating the Efficacy of Transcatheter Arterial Chemoembolization for Hepatocellular Carcinoma. OncoTargets and Therapy, 9, 7029-7037. https://doi.org/10.2147/OTT.S112555

[11] Bonekamp, S., Jolepalem, P., Lazo, M., et al. (2011) Hepatocellular Carcinoma: Response to TACE Assessed with Semiautomated Volumetric and Functional Analysis of Diffusion-Weighted and Contrast-Enhanced MR Imaging Data. Radiology, 260, 752-761. https://doi.org/10.1148/radiol.11102330

[12] Yu, J.S., Kim, J.H., Chung, J.J., et al. (2009) Added Value of Diffusion-Weighted Imaging in the MRI Assessment of Perilesional Tumor Recurrence after Chemoembolization of Hepatocellular Carcinomas. Journal of Magnetic Resonance 
Imaging, 30, 153-160. https://doi.org/10.1002/jmri.21818

[13] Mannelli, L., Kim, S., Hajdu, C.H., et al. (2013) Serial Diffusion-Weighted MRI in Patients with Hepatocellular Carcinoma: Prediction and Assessment of Response to Transarterial Chemoembolization. Preliminary Experience. European Journal of Radiology, 82, 577-582. https://doi.org/10.1016/j.ejrad.2012.11.026

[14] Jiang, Z.X., Peng, W.J., Li, W.T., et al. (2008) Effect of b Value on Monitoring Therapeutic Response by Diffusion-Weighted Imaging. World Journal of Gastroenterology, 14, 5893-5899. https://doi.org/10.3748/wjg.14.5893

[15] Le Bihan, D., Breton, E., Lallemand, D., et al. (1986) MR Imaging of Intravoxel Incoherent Motions: Application to Diffusion and Perfusion in Neurologic Disorders. Radiology, 161, 401-407. https://doi.org/10.1148/radiology.161.2.3763909

[16] Watanabe, H., Kanematsu, M., Goshima, S., et al. (2014) Characterizing Focal Hepatic Lesions by Free-Breathing Intravoxel Incoherent Motion MRI at 3.0 T. Acta Radiologica, 55, 1166-1173. https://doi.org/10.1177/0284185113514966

[17] Woo, S., Lee, J.M., Yoon, J.H., et al. (2014) Intravoxel Incoherent Motion Diffusion-Weighted MR Imaging of Hepatocellular Carcinoma: Correlation with Enhancement Degree and histologic Grade. Radiology, 270, 758-767. https://doi.org/10.1148/radiol.13130444

[18] Xiao, Y., Pan, J., Chen, Y., et al. (2015) Intravoxel Incoherent Motion-Magnetic Resonance Imaging as an Early Predictor of Treatment Response to Neoadjuvant Chemotherapy in Locoregionally Advanced Nasopharyngeal Carcinoma. Medicine (Baltimore), 94, e973. https://doi.org/10.1097/MD.0000000000000973

[19] Hauser, T., Essig, M., Jensen, A., et al. (2014) Prediction of Treatment Response in Head and Neck Carcinomas Using IVIM-DWI: Evaluation of Lymph Node Metastasis. European Journal of Radiology, 83, 783-787. https://doi.org/10.1016/j.ejrad.2014.02.013

[20] Hauser, T., Essig, M., Jensen, A., et al. (2013) Characterization and Therapy Monitoring of Head and Neck Carcinomas Using Diffusion-Imaging-Based Intravoxel Incoherent Motion Parameters-Preliminary Results. Neuroradiology, 55, 527-536. https://doi.org/10.1007/s00234-013-1154-9

[21] Hu, Y.C., Yan, L.F., Wu, L., et al. (2014) Intravoxel Incoherent Motion Diffusion-Weighted MR Imaging of Gliomas: Efficacy in Preoperative Grading. Scientific Reports, 4, Article No. 7208. https://doi.org/10.1038/srep07208

[22] Che, S., Zhao, X., Ou, Y., et al. (2016) Role of the Intravoxel Incoherent Motion Diffusion Weighted Imaging in the Pre-Treatment Prediction and Early Response Monitoring to Neoadjuvant Chemotherapy in Locally Advanced Breast Cancer. Medicine, 95, e2420. https://doi.org/10.1097/MD.0000000000002420

[23] Deng, Y., Yang, B., Peng, Y., et al. (2018) Use of Intravoxel Incoherent Motion Diffusion-Weighted Imaging to Detect Early Changes in Diabetic Kidneys. Abdominal Radiology, 43, 2728-2733. https://doi.org/10.1007/s00261-018-1521-4

[24] Lv, J., Huang, W., Zhang, J., et al. (2018) Performance of U-Net Based Pyramidal Lucas-Kanade Registration on Free-Breathing Multi-b-Value Diffusion MRI of the Kidney. The British Journal of Radiology, 91, Article ID: 20170813. https://doi.org/10.1259/bjr.20170813

[25] Feng, Y.Z., Chen, X.Q., Yu, J., et al. (2018) Intravoxel Incoherent Motion (IVIM) at 3.0 T: Evaluation of Early Renal Function Changes in Type 2 Diabetic Patients. $A b$ dominal Radiology, 43, 2764-2773. https://doi.org/10.1007/s00261-018-1555-7

[26] Park, S., Yoon, J.K., Chung, N.S., et al. (2018) Correlations between Intravoxel Incoherent Motion Diffusion-Weighted MR Imaging Parameters and 18F-FDG 
PET/CT Metabolic Parameters in Patients with Vertebral Bone Metastases: Initial Experience. The British Journal of Radiology, 91, Article ID: 20170889. https://doi.org/10.1259/bjr.20170889

[27] Liu, K., Yang, L., Zhang, X.M., et al. (2017) HIF-1 $\alpha$ and VEGF Levels for Monitoring Hepatocellular Carcinoma Treatment Response to Transcatheter Arterial Chemoembolization. Translational Cancer Research, 6, 1043-1049. https://doi.org/10.21037/tcr.2017.08.32

[28] Min, X.L., Yang, L., Zhang, X.M., et al. (2017) Changes of Tryptase in Patients with Hepatocellular Carcinoma after Transarterial Chemoembolization. Translational Cancer Research, 6, 1061-1067. https://doi.org/10.21037/tcr.2017.08.43

[29] Liu, K., Min, X.L., Peng, J., et al. (2016) The Changes of HIF-1alpha and VEGF Expression after TACE in Patients with Hepatocellular Carcinoma. Journal of Clinical Medicine Research, 8, 297-302. https://doi.org/10.14740/jocmr2496w

[30] Colli, A., Fraquelli, M., Casazza, G., et al. (2006) Accuracy of Ultrasonography, Spiral CT, Magnetic Resonance, and Alpha-Fetoprotein in Diagnosing Hepatocellular Carcinoma: A Systematic Review. American Journal of Gastroenterology, 101, 513-523. https://doi.org/10.1111/j.1572-0241.2006.00467.x

[31] Yang, K., Zhang, X.M., Yang, L., et al. (2016) Advanced Imaging Techniques in the Therapeutic Response of Transarterial Chemoembolization for Hepatocellular Carcinoma. World Journal of Gastroenterology, 22, 4835-4847.

https://doi.org/10.3748/wjg.v22.i20.4835

[32] Loffroy, R., Favelier, S., Cherblanc, V., et al. (2013) C-Arm Dual-Phase Cone-Beam CT: A Revolutionary Real-Time Imaging Modality to Assess Drug-Eluting Beads TACE Success in Liver Cancer Patients. Quantitative Imaging in Medicine and Surgery, 3, 196-199.

[33] Lencioni, R., Caramella, D. and Bartolozzi, C. (1995) Hepatocellular Carcinoma: Use of Color Doppler US to Evaluate Response to Treatment with Percutaneous Ethanol Injection. Radiology, 194, 113-118. https://doi.org/10.1148/radiology.194.1.7997536

[34] Bartolozzi, C., Lencioni, R., Caramella, D., et al. (1994) Hepatocellular Carcinoma: $\mathrm{CT}$ and MR Features after Transcatheter Arterial Embolization and Percutaneous Ethanol Injection. Radiology, 191, 123-128. https://doi.org/10.1148/radiology.191.1.8134557

[35] Jiang, T., Zhu, A.X. and Sahani, D.V. (2013) Established and Novel Imaging Biomarkers for Assessing Response to Therapy in Hepatocellular Carcinoma. Journal of Hepatology, 58, 169-177. https://doi.org/10.1016/j.jhep.2012.08.022

[36] Park, Y.S., Lee, C.H., Kim, J.H., et al. (2014) Using Intravoxel Incoherent Motion (IVIM) MR Imaging to Predict Lipiodol Uptake in Patients with Hepatocellular Carcinoma Following Transcatheter Arterial Chemoembolization: A Preliminary Result. Magnetic Resonance Imaging, 32, 638-646. https://doi.org/10.1016/j.mri.2014.03.003

[37] $\mathrm{Wu}, \mathrm{L} ., \mathrm{Xu}, \mathrm{P} ., \mathrm{Rao}, \mathrm{S}$., et al. (2017) ADCtotal Ratio and D Ratio Derived from Intravoxel Incoherent Motion Early after TACE Are Independent Predictors for Survival in Hepatocellular Carcinoma. Journal of Magnetic Resonance Imaging, 46, 820-830. https://doi.org/10.1002/jmri.25617

[38] Mazaheri, Y., Vargas, H.A., Akin, O., et al. (2012) Reducing the Influence of b-Value Selection on Diffusion-Weighted Imaging of the Prostate: Evaluation of a Revised Monoexponential Model within a Clinical Setting. Journal of Magnetic Resonance Imaging, 35, 660-668. https://doi.org/10.1002/jmri.22888 
[39] Döpfert, J., Lemke, A., Weidner, A., et al. (2011) Investigation of Prostate Cancer Using Diffusion-Weighted Intravoxel Incoherent Motion Imaging. Magnetic Resonance Imaging, 29, 1053-1058. https://doi.org/10.1016/j.mri.2011.06.001 